\title{
DISTRIBUTION OF HOLES AND TEARS IN PRIMARY RETINAL DETACHMENT*
}

\author{
BY \\ C. GRANT TULLOH \\ Bristol Eye Hospital
}

THE presence of holes in detached retinae was noted shortly after ophthalmoscopic examination became possible (Coccius, 1853; von Graefe, 1863a and b; Liebreich, $1863 ; 1885)$, and relatively soon thereafter de Wecker and de Jaeger (1870) drew attention to their striking frequency.

Von Graefe originally thought that they were part of the healing process, until de Wecker and de Jaeger suggested that they were causative. The dramatic therapeutic results obtained by Gonin on closing such holes focused attention on them anew, and it was his work from 1923 onwards that established their importance in the aetiology and treatment of detachment. He found holes in 85 per cent. of cases (1930), Arruga (1932) in 90 per cent., and Weve (1932) also in 90 per cent.

Gonin's work was supported at about the same time by the observations of Lister (1924), Brons (1924), Vogt (1929), and Kümmell (1929). On the other hand, this view was at first (and still is) objected to on the grounds that, experimentally and clinically, holes may heal and the detachment persist, detachments may occur without holes, and holes without detachments. It would seem, therefore, that in some cases the presence of a hole is not the sole cause of the detachment, and that detachment does not invariably result from the presence of a hole.

In general, it is thought that retinal holes are primarily consequent on retinal changes, while retinal tears are primarily due to vitreous changes.

With regard to the role of retinal changes, there can be little doubt that an inherent weakness of the retina at the ora, or the presence of a congenital retinal cyst, is the predisposing cause of most anterior dialyses occurring in young people. A dialysis may be precipitated by minor trauma.

The mechanism of production of round retinal holes, however, is less well understood. Most authors are agreed that there is choroidoretinal degeneration (Bagley, 1948; Sen, 1948; Shipman and Luce, 1954; Adams, 1956), but the cause of this is not clear. The two main schools of thought are, on the one hand, that degeneration occurs as a result of vascular inadequacy, leading to cystic changes at the periphery of the retina and associated with senility, premature or otherwise, or myopia (Knapp, 1943; Schulte, 1948; Bonavolontà, 1953; Michaelson, 1954; Alfonso, 1957). On the 
other hand, some believe that small foci of old choroidoretinitis of indeterminate aetiology are the principal factor (Arruga, 1929, 1932, 1933, 1934; Duke-Elder, 1945; Longhena, 1950).

In addition, it is probable that commotio retinae resulting from direct trauma can produce delayed hole formation and detachment.

With regard to myopic detachments, Arkin (1948) has suggested that it is an increase in the size of the retina (and not the sclera) which is the primary occurrence, a view with which Vail (1948) disagrees, pointing out the frequency with which equatorial scleral staphylomata occur in myopia.

Vitreous changes are thought to play a major part in the mechanism, particularly of those cases with a retinal tear or hole and an operculum attached to a vitreous band. It is suggested that as a result of inflammatory or degenerative retinal changes the vitreous becomes firmly adherent to this tissue at the affected point. Later the vitreous as a whole becomes detached or retracted forwards, and the adhesion tears a hole in the retina. This view is supported by the work of Böck (1949), Posner (1951; 1952), Wadsworth (1952), Hruby (1953; 1955), Adams (1956), and Frey (1956).

Teng and Chi (1957) consider that the most potent factor in retinal detachment is liquefaction of the posterior vitreous, and forward retraction of the anterior vitreous, which is adherent to the retina in the region of the equator, either at the site of a congenital rosette or as a result of senile peripheral cystic degeneration. Agarwal (1956) has advanced the view that a primary vitreous degeneration produces an inflammatory reaction in the retina and choroid resulting in hole formation.

Adamantiadis and Rangavi (1950), however, have thrown doubt on the vitreous theory by following up 26 cases of vitreous detachment for three to four years, and in only one, a high myope, did retinal detachment develop.

Some writers have suggested that the pull of the extra-ocular muscles is a contributory factor in the production of retinal tears, pointing out the frequency with which tears occur close to the insertion of the superior oblique muscle (Campos, 1947; Krümmel, 1951; Garkavi, 1952; Stagni, 1953). There is little evidence on which to base these assertions.

The observations of Shapland (1932) regarding the meridional distribution of retinal perforations form the basis of the present investigations, and his results will be compared with those obtained in the present series by the use of newer methods.

The radial distribution of retinal perforations has not been analysed hitherto, so this aspect of their distribution is also investigated.

\section{Material}

The material used in these investigations consisted of cases of retinal detachment referred to the Detachment Clinic at Moorfields Eye Hospital, London, to which clinic cases are normally referred mainly from the Eye Hospital itself, but also from other London and provincial hospitals. They comprise routine detachments for full pre-operative investigation, post-operative detachments, detachments in which the fundus is difficult to see owing to opacities in the media, and detachments in which no retinal hole can be seen by ordinary methods.

Only pre-operative detachments in which holes or tears could be seen and the fundus fully visualized were included in the investigations. All the cases were examined by the author. 


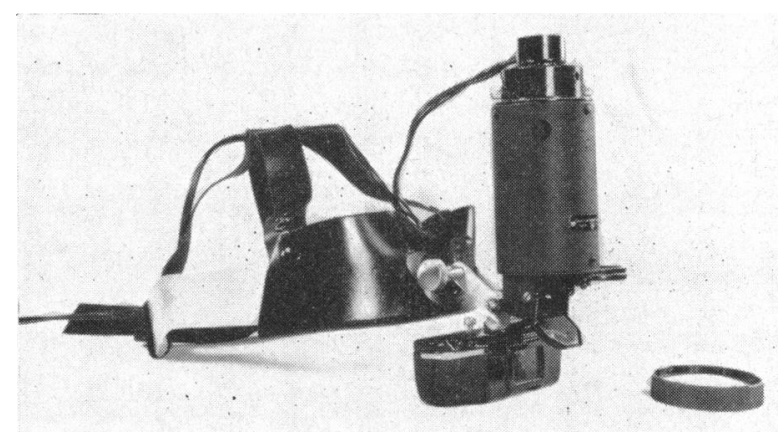

FIG. 1.-Schepen's binocular indirect ophthalmoscope.

\section{Methods}

All detachments were examined with the Schepens binocular indirect ophthalmoscope (Fig. 1). The advantages of this method over uniocular direct ophthalmoscopy are:

(1) A binocular stereoscopic view is obtained.

(2) The smaller magnification $(x 5)$ which is achieved gives a better overall view of the fundus, showing sharper contrast between features such as holes and haemorrhages and intact retina. This reduces the likelihood of missing important details, and enables a diagram to be made of the whole fundus in greater comfort and more speedily; such a diagram was made in all cases.

(3) Brighter illumination is possible, allowing an adequate view through hazy media and also of the periphery because only a small portion of the available light is utilized at very oblique angles of observation.

The greater magnification obtained with direct ophthalmoscopy $(\times 15)$ was necessary occasionally where doubtful lesions were discovered. In addition, Trantas's manœuvre was utilized when doubtful lesions were seen near the ora.

\section{Meridional Distribution of Holes and Tears}

From the fundus diagrams obtained holes and tears were appropriately allocated to each of the four quadrants of the retina-upper and lower nasal and upper and lower temporal. The numbers of holes and tears were then analysed and compared statistically.

\section{Radial Distribution of Holes and Tears}

The diagrams constructed by the technique described were of uniform size, and lesions depicted thereon were allocated to retinal areas of equal width from the macula to the ora according to the following method. A scale drawing of a schematic eye was prepared with the following measurements:

\begin{tabular}{lr} 
Radius of cornea-anterior surface & $7.86 \mathrm{~mm}$. \\
Radius of lens-anterior surface & $11.00 \mathrm{~mm}$. \\
\multicolumn{1}{c}{ posterior surface } & $5.78 \mathrm{~mm}$. \\
Radius of retina & $11.06 \mathrm{~mm}$. \\
Radius of sclera-transverse & $12.07 \mathrm{~mm}$. \\
Centre of curvature of retina from anterior corneal surface & $11.95 \mathrm{~mm}$. \\
Centre of curvature of anterior sclera & $13.1 \mathrm{~mm}$. \\
Centre of curvature of posterior sclera & $12.09 \mathrm{~mm}$. \\
Thickness of lens & $3.62 \mathrm{~mm}$. \\
Anterior corneal surface to anterior surface of lens & $3.84 \mathrm{~mm}$. \\
Index refraction of cornea, aqueous, and vitreous & 1.336 \\
Index refraction of lens & 1.43
\end{tabular}


By means of ray tracing (Fig. 2) and appropriate calculations, the observer's estimated projection of equal retinal widths from macula to ora on to a flat surface was obtained.

The surface of a retinal diagram, corresponding exactly in size to the diagrams already prepared, was then divided into seven areas from ora to macula in the appropriate ratios corresponding to equal retinal widths (Fig. 3). Holes or tears from each retinal diagram

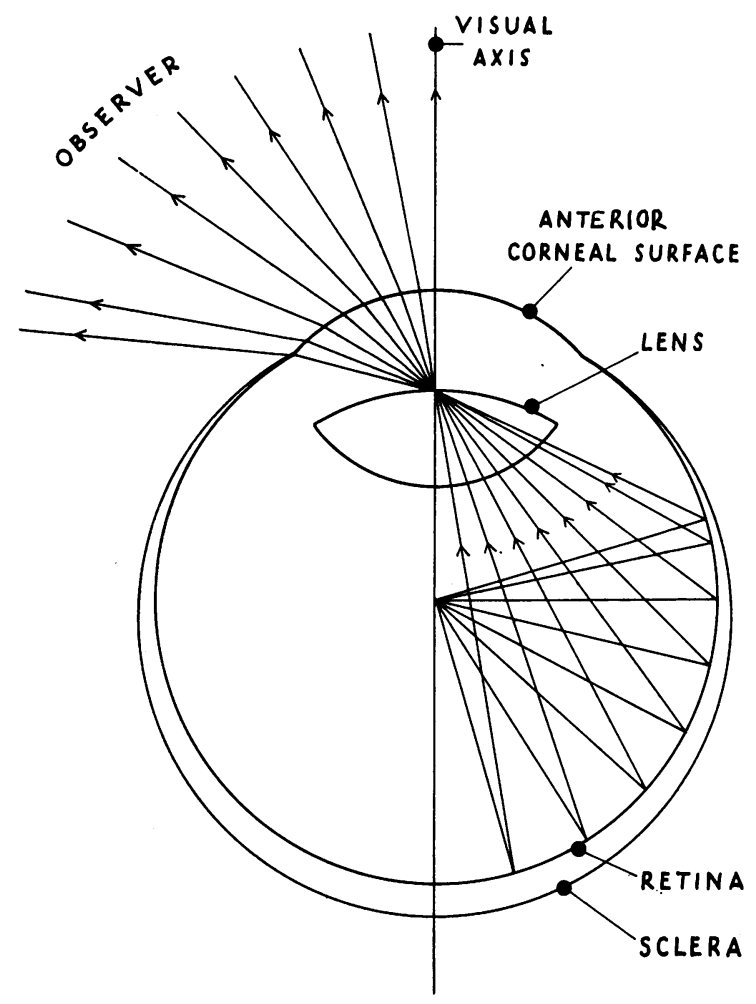

FIG. 2.-Observer's projection of equal retinal widths as determined by ray tracing.

FIG. 3.- Retinal diagram divided into seven areas of equal retinal width from ora to macula.

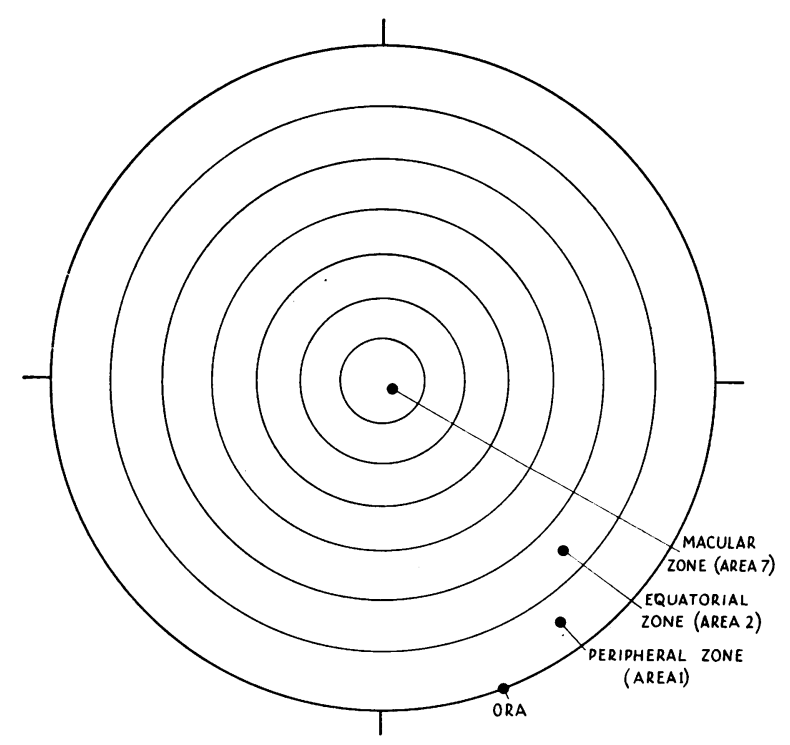


were allocated to the areas obtained. Owing to the gradually changing radii, and therefore circumferences of these areas, the results obtained do not reflect the true relative radial distribution of retinal holes. The average circumference of each area was therefore calculated, and the number of holes for areas of equal circumference estimated.

These figures were then analysed and compared statistically.

\section{Results}

\section{Meridional Distribution of Holes and Tears}

The 422 cases of retinal detachment ( 236 males and 186 females) were examined and retinal holes and tears found therein divided into four groups: round holes, arrow-head tears, slit tears, and anterior dialyses.

The cases were further divided into four classes according to their refraction: hypermetropes and emmetropes, low myopes $(-0.25$ to $-6.00 \mathrm{D})$, high myopes (over $-6.00 \mathrm{D}$ ), and aphakics.

Round Holes. - A total of 172 round holes was seen in 57 non-myopes ( 34 males, 23 females). Macular holes were excluded. Fig. 4 shows their distribution in the four retinal quadrants as follows: Upper temporal quadrant, 36.05 per cent. Lower temporal quadrant, $40 \cdot 70$ per cent. Upper nasal quadrant, 9.88 per cent. Lower nasal quadrant, 13.37 per cent. Holes thus occur most frequently in the lower temporal quadrant and least frequently in the upper nasal quadrant. Their preponderance on the temporal side can be demonstrated, for the ratio temporal: nasal is $132: 40$ which is statistically significant $\left(\chi^{2}=49 \cdot 21, \mathrm{~N}=1, \mathrm{P}<0.001\right)$. There is, however, no significant difference between the number of holes in the upper half (79) and that in the lower half of the retina (93), although there is a slight preponderance of holes below $\left(\chi^{2}=1 \cdot 14, N=1, P<0.50>0 \cdot 10\right)$.

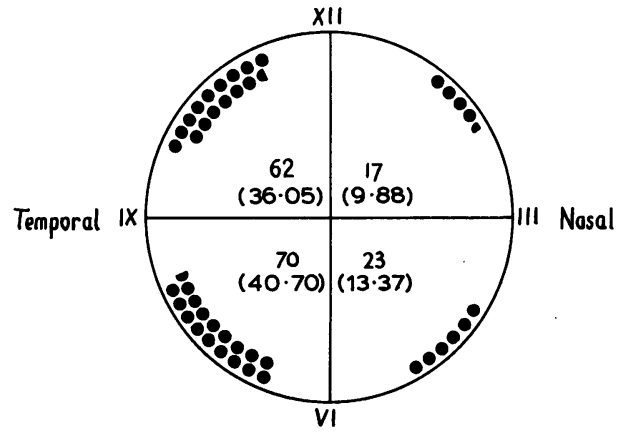

Fig. 4.-Meridional distribution of 172 round holes in 57 non-myopic eyes (percentages in brackets).

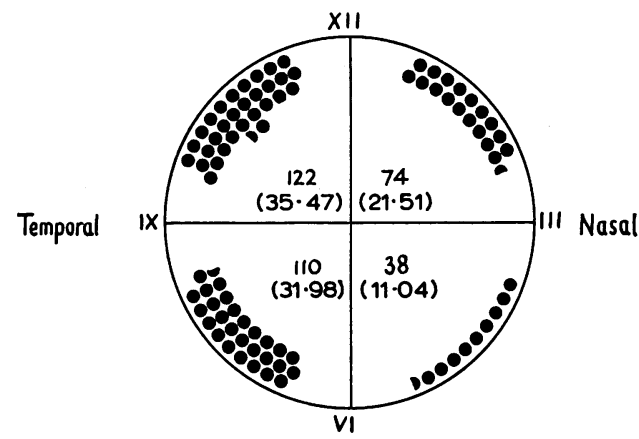

FIG. 5.-Meridional distribution of 344 round holes in 128 myopic eyes (percentages in brackets).

In myopes the figures for the respective quadrants are slightly different. A total of 344 holes was seen in 128 eyes ( 75 males, 53 females). Fig. 5 shows their distribution in the four retinal quadrants as follows, macular holes again being excluded: Upper temporal quadrant, 35.47 per cent. Lower temporal quadrant, 31.98 per cent. Upper nasal quadrant, 21.51 per cent. Lower nasal quadrant, 11.04 per cent. 
TABLE I

Distribution of Round Holes in Myopes and Non-myopes

\begin{tabular}{l|c|c|c}
\hline & $\begin{array}{c}\text { Upper Half } \\
\text { of Retina }\end{array}$ & $\begin{array}{c}\text { Lower Half } \\
\text { of Retina }\end{array}$ & Total \\
\cline { 2 - 3 } Myopes & 196 & 148 & 344 \\
Non-Myopes & 79 & 93 & 172 \\
\hline Total & 275 & 241 & 516 \\
\hline
\end{tabular}

Holes thus occur most frequently in the upper temporal quadrant and least frequently in the lower nasal quadrant in myopes. There is the same preponderance on the temporal side as in the case of the non-myopes, the ratio temporal : nasal being 232 : 112 which is statistically significant $\left(\chi^{2}=41 \cdot 86, \mathrm{~N}=1, \mathrm{P}<0.001\right)$.

The larger proportion of holes in the upper nasal quadrant is reflected in the ratio between the number of holes in the upper half (196) to that in the lower half of the retina (148). This difference is statistically significant $\left(\chi^{2}=6 \cdot 70, \mathrm{~N}=1, \mathrm{P}<0.01\right.$ $>0.001)$. In view of the fact that this ratio is the reverse of that found in nonmyopes, the two ratios were compared statistically (Table I).

A $\chi^{2}$ test showed a significant difference between the distribution of holes in the two refractive groups $\left(\chi^{2}=5.62, \mathrm{~N}=1, \mathrm{P}<0.02>0.01\right)$.

With regard to the age and sex distribution in the two groups, the mean age of the males was $43.56 \pm 15.47$ years ( 75 cases) in the myopic series, and $41.00 \pm 18.39$ years (34 cases) in the non-myopic series. A $t$ test showed no significant difference between the two $(t=0 \cdot 76, \mathrm{~N}=107, \mathrm{P}>0 \cdot 10)$. The mean age of the females was $48.75 \pm 13.77$ years (53 cases) in the first series, and $55.74 \pm 17.76$ years ( 23 cases) in the second. A $t$ test showed no significant difference between the two $(t=1 \cdot 86$, $\mathrm{N}=74, \mathrm{P}<0 \cdot 10>0 \cdot 05$ ).

The sex distribution is shown in Table II.

A $\chi^{2}$ test on these figures showed no significant difference between the series $\left(\chi^{2}=0.018, \mathrm{~N}=1, \mathrm{P}<0.90>0.50\right)$.

TABLE II

Sex Distribution of Myopes and Non-myopes Presenting with Round Retinal Holes

\begin{tabular}{l|c|c|c}
\hline & Males & Females & Total \\
\hline Myopes & 75 & 53 & 128 \\
Non-Myopes & 34 & 23 & 57 \\
\hline Total & 109 & 76 & 185 \\
\hline
\end{tabular}

Arrow-head Tears. - A total of 37 arrow-head tears was observed in 35 nonmyopes (18 males, 17 females) and their meridional distribution is shown in Fig. 6 as follows: Upper temporal quadrant, 40.54 per cent. Lower temporal quadrant, 24.33 per cent. Upper nasal quadrant, 32.43 per cent. Lower nasal quadrant, 2.70 per cent. Tears thus occur most frequently in the upper temporal quadrant and rarely in the lower nasal quadrant. Their predilection for the upper retina can be demonstrated, the ratio upper retina : lower retina being $27: 10$ and this difference is statistically significant $\left(\chi^{2}=6.92, \mathrm{~N}=1, \mathrm{P}<0 \cdot 01>0 \cdot 001\right)$. On the other 
hand, the ratio temporal retina : nasal retina is $24: 13$ and this difference is not statistically significant $\left(\chi^{2}=2 \cdot 70, \mathrm{~N}=1, \mathrm{P}<0.50>0.10\right)$. Possibly larger numbers might show a significant difference.

In myopes, a total of 185 arrow-head tears was observed in 130 eyes $(67$ males, 63 females) and their meridional distribution is shown in Fig. 7 as follows: Upper temporal quadrant, 40.54 per cent. Lower temporal quadrant, 20.00 per cent. Upper nasal quadrant, 25.41 per cent. Lower nasal quadrant, 14.05 per cent. Thus these tears favour the upper temporal quadrant and least commonly occur in the lower nasal quadrant. Their preponderance in the upper half of the retina can be demonstrated, the ratio upper retina : lower retina being $122: 63$ and this difference is statistically significant $\left(\chi^{2}=18.82, \mathrm{~N}=1, \mathrm{P}<0.001\right)$. Further, it is evident that the temporal retina is the more often affected, the ratio temporal retina : nasal retina being $112: 73$ and this difference is statistically significant $\left(\chi^{2}=8 \cdot 22\right.$, $\mathrm{N}=1, \mathrm{P}<0.01>0.001$ ).

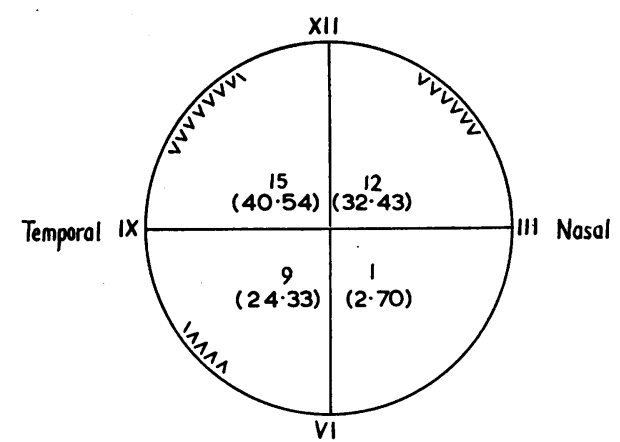

FIG. 6.-Meridional distribution of 37 arrowhead tears in 35 non-myopic eyes (percentages in brackets).

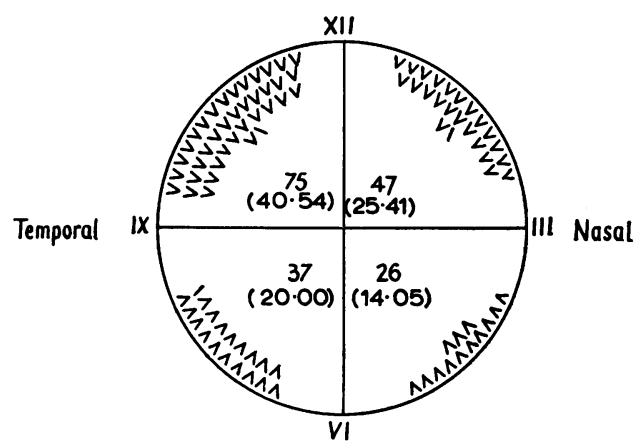

FIG. 7.-Meridional distribution of 185 arrowhead tears in 130 myopic eyes (percentages in brackets).

Slit Tears.-A total of 6 slit tears was seen in 6 non-myopes (6 males), but they are rarely seen and their numbers are of no significance.

In myopes, a total of 35 such tears was observed in 23 eyes ( 14 males, 9 females) and their meridional distribution was as follows: Upper temporal quadrant, 28.57 per cent. Lower temporal quadrant, 34.29 per cent. Upper nasal quadrant, 31.43 per cent. Lower nasal quadrant, 5.71 per cent. Thus these lesions are rare in the lower nasal quadrant but are distributed fairly evenly elsewhere. They are of little significance on account of their scarcity.

Dialyses.-Forty-six dialyses were observed in 43 eyes ( 29 males, 14 females) of which 32 were non-myopic and 11 myopic (in 4 instances more than 6 dioptres). Their meridional distribution is shown in Fig. 8 as follows: Upper temporal quadrant, 10.87 per cent. Lower temporal quadrant, 67.39 per cent. Upper nasal quadrant, 2.17 per cent. Lower nasal quadrant, 19.57 per cent. The preponderance of such lesions in the lower temporal quadrant is evident and statistical analysis confirms that the distribution varies significantly from the mean number of dialyses per quadrant $(11 \cdot 5)\left(\chi^{2}=46 \cdot 87, \mathrm{~N}=3, \mathrm{P}<0 \cdot 001\right)$. 


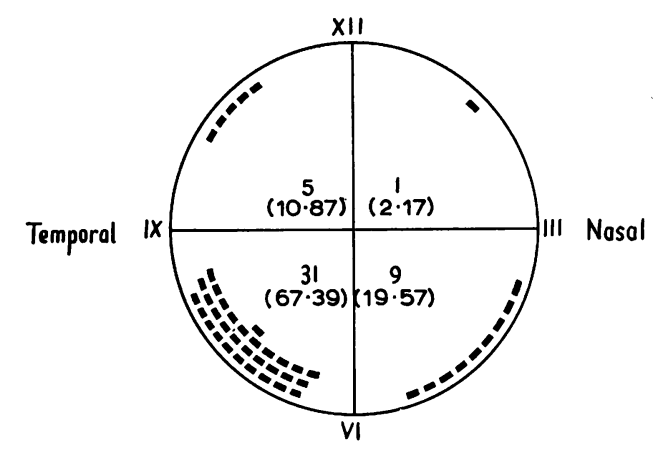

FIG. 8.-Meridional distribution of 46 dialyses in 43 eyes (percentages in brackets).

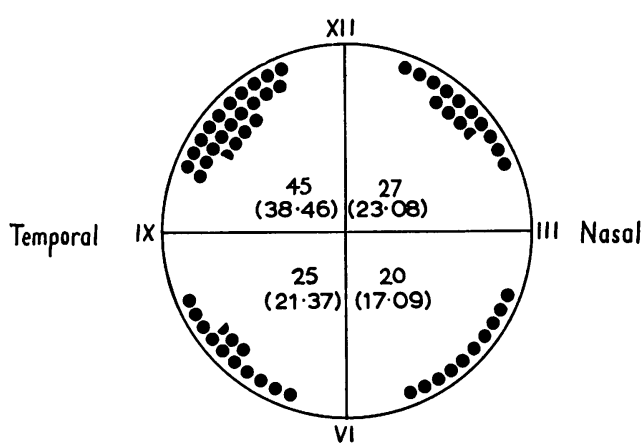

Fig. 9.-Meridional distribution of 117 holes and tears in 49 eyes (percentages in brackets).

Aphakia.-A total of 117 retinal perforations was observed in 49 eyes (24 males, 25 females) of which 14 were myopic. The meridional distribution of these lesions is shown in Fig. 9 as follows: Upper temporal quadrant, 38.46 per cent. Lower temporal quadrant, 21.37 per cent. Upper nasal quadrant, 23.08 per cent. Lower nasal quadrant, 17.09 per cent. Thus they favour the upper temporal quadrant and least commonly occur in the lower nasal quadrant. Their predilection for the upper half of the retina can be demonstrated, the ratio upper retina : lower retina being $72: 43$ and this difference is statistically significant $\left(\chi^{2}=6 \cdot 23, \mathrm{~N}=1\right.$, $\mathrm{P}<0.02>0.01)$. Further, there is a preponderance of perforations on the temporal side, the ratio temporal retina : nasal retina being $70: 47$ and this difference is also statistically significant $\left(\chi^{2}=4.52, \mathrm{~N}=1, \mathrm{P}<0.05>0.02\right)$.

\section{Radial Distribution of Holes and Tears}

The 451 cases ( 252 males, 199 females) were examined and retinal holes and tears found therein divided into three groups: round holes, arrow-head tears, and slit tears.

The cases were further divided into three classes according to their refraction: hypermetropes and emmetropes, low myopes $(-0.25$ to $-6.00 \mathrm{D})$, and high myopes (over $-6 \cdot 00 \mathrm{D}$ ).

Round Holes.-A total of 165 such holes was seen in 57 non-myopes (34 males, 23 females). The estimated number of holes for each of the seven areas from ora to macula is shown in Fig. 10 as follows:

$\begin{array}{lccccccc}\text { Area } & 1 & 2 & 3 & 4 & 5 & 6 & 7 \\ \text { Number of holes (estimated) } & 42 & 108 & 22 \cdot 4 & 18 \cdot 9 & 11 \cdot 6 & 15 \cdot 3 & 46 \cdot 2\end{array}$

It will be seen that by far the largest number of holes (108) occurs in Area 2 (the equatorial zone). This figure was compared statistically with that for Area 1 (the peripheral zone) (42) and the difference found to be significant $\left(\chi^{2}=28 \cdot 9, \mathrm{~N}=1\right.$, $P<0.001)$. Thus these holes occur preferentially in the equatorial and not the peripheral zone.

Area 7 (the macular region) also develops a considerable number of holes. Indeed, more holes occur here (for the size of the area) than anywhere else except the equatorial zone. 


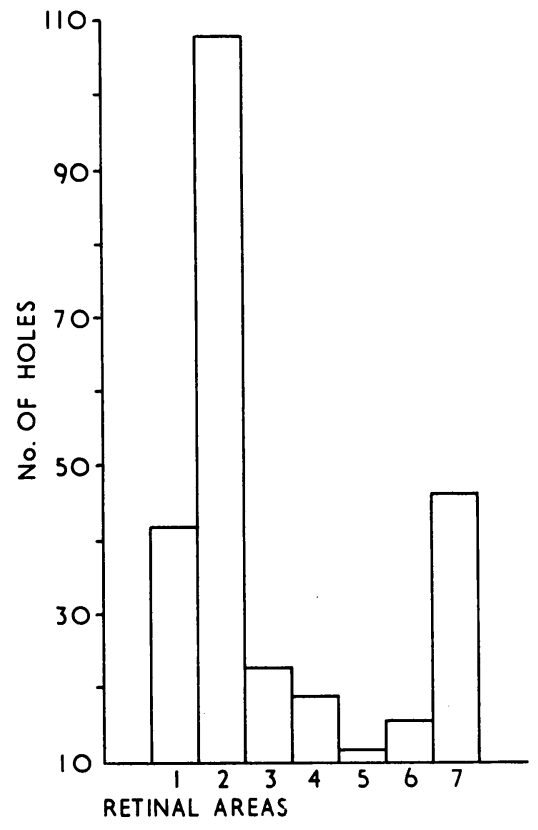

FIG. 10.-Radial distribution of round holes in 57 non-myopes. Note: The numbers of holes and tears shown in Figs. 10-13 are estimated (see text).

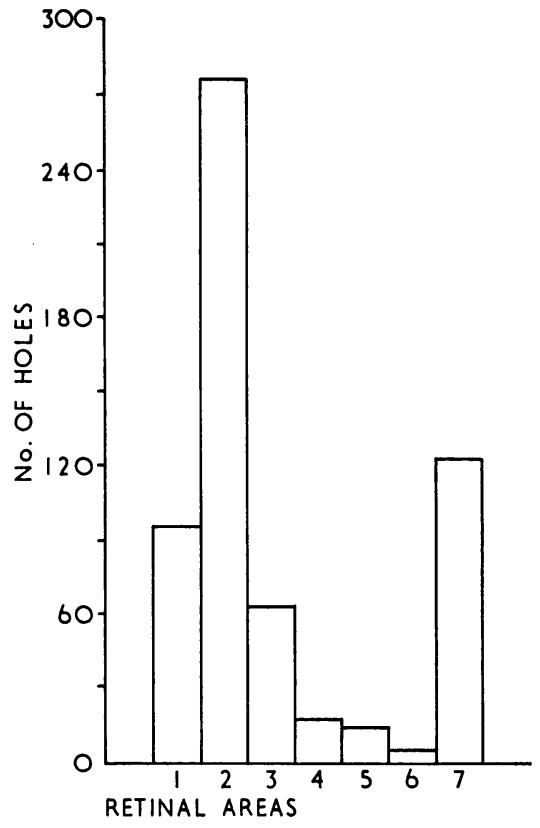

FIG. 11.- Radial distribution of round holes in 148 myopes.

In 39 instances multiple holes were present and the estimated number of instances in the respective areas demonstrates again that the largest number occurs in Area 2 $(25 \cdot 2)$, thus:
Area $1 \quad 2 \quad 3$ $3+8$ $\begin{array}{lccc}4 & 5 & 6 & 7\end{array}$
$\begin{array}{llllllll}\text { Number of instances (estimated) } & 10 & 25 \cdot 2 & 4 \cdot 8 & 6 \cdot 3 & 2 \cdot 9 & 5 \cdot 1 & 0\end{array}$

Multiple macular holes were never seen.

In myopes a total of 389 holes was seen in 148 eyes ( 87 males, 61 females) and the estimated number of holes for each of the 7 areas is shown in Fig. 11 as follows:

$\begin{array}{lccccccc}\text { Area } & 1 & 2 & 3 & 4 & 5 & 6 & 7 \\ \text { Number of holes (estimated) } & 96 & 276 & 64 & 18 \cdot 9 & 14 \cdot 5 & 5 \cdot 1 & 123 \cdot 2\end{array}$

It will be seen that by far the largest number of holes (276) occurs in Area 2 (the equatorial zone). This figure was compared statistically with that for Area 1 (the peripheral zone) (96) and the difference found to be significant $\left(\chi^{2}=87 \cdot 10, \mathrm{~N}=1\right.$, $\mathrm{P}<0.001)$. Thus these holes also occur preferentially in the equatorial and not the peripheral zone.

Area 7 (the macular region) also develops a considerable number of holes, and again, more holes occur here (for the size of the area) than anywhere else except the equatorial zone.

In 83 instances multiple holes were present and the estimated number of instances in the respective areas demonstrates again that the largest number occurs in Area 2 


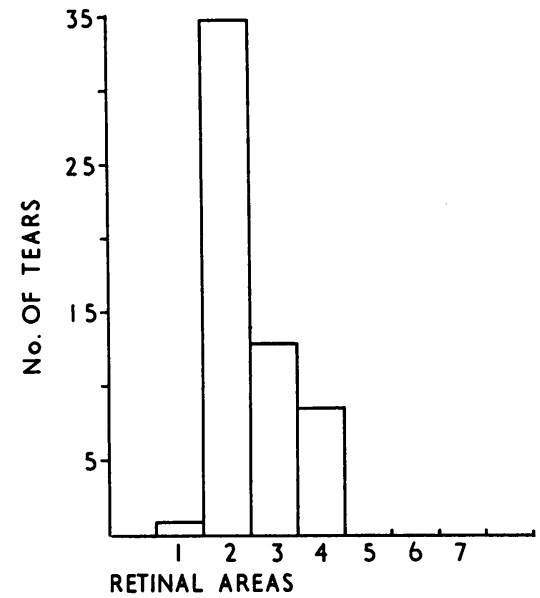

FIG. 12.-Radial distribution of arrowhead tears in 39 non-myopes.

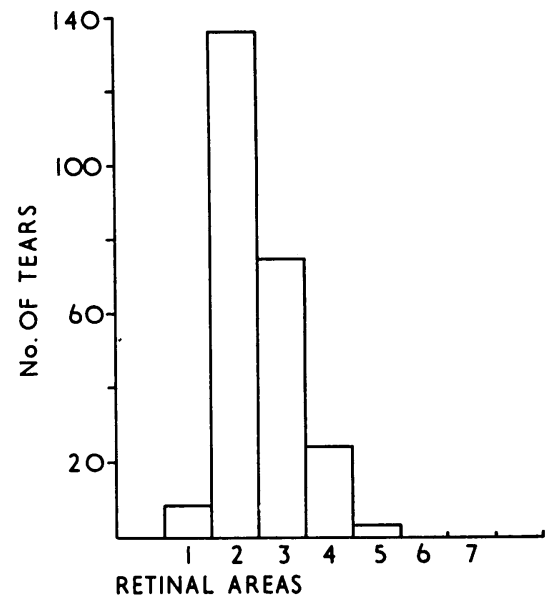

FIG. 13.- Radial distribution of arrowhead tears in 127 myopes.

$(57 \cdot 6)$, thus:

\section{Area \\ Number of instances (estimated)

Multiple macular holes were never seen.

Arrow-head Tears. - A total of 42 arrow-head tears was observed in 39 nonmyopes (20 males, 19 females). The estimated number of tears for each of the seven areas from ora to macula is shown in Fig. 12 as follows:
Area

2

3
4

$\begin{array}{lll}5 & 6 & 7\end{array}$
$\begin{array}{llllllll}\text { Number of tears (estimated) } & 1 & 34.8 & 12 \cdot 8 & 8 \cdot 4 & 0 & 0 & 0\end{array}$

It will be seen that the largest number (34.8) occurs in Area 2 (the equatorial zone). This figure was compared statistically with that for Area 3 (12.8), and not with that for Area 1 as heretofore, since such lesions rarely occur in the latter area (a matter which will be discussed later). The difference between the two figures was found to be significant $\left(\chi^{2}=9 \cdot 26, \mathrm{~N}=1, \mathrm{P}<0.01>0.001\right)$. Thus arrowhead tears occur preferentially in the equatorial zone.

In myopes a total of 181 tears was observed in 127 eyes (66 males, 61 females). The estimated number of tears for each of the seven areas is shown in Fig. 13 as follows:

$$
\begin{aligned}
& \begin{array}{llllllll}
\text { Area } & 1 & 2 & 3 & 4 & 5 & 6 & 7
\end{array} \\
& \begin{array}{llllllll}
\text { Number of tears (estimated) } & 9 & 136.8 & 73.6 & 23 \cdot 1 & 2.9 & 0 & 0
\end{array}
\end{aligned}
$$

It will be seen that the largest number (136.8) occurs in Area 2 (the equatorial zone).

This figure was compared statistically with that for Area $3(73.6)$ as in the case of the non-myopes, and the difference between the two found to be significant $\left(\chi^{2}=\right.$ $18.90, \mathrm{~N}=1, \mathrm{P}<0.001)$.

Thus, as in the case of the non-myopes, in myopes also these tears favour the equatorial zone.

By contrast with round holes, arrow-head tears were never seen in the macular area, in either non-myopes or myopes. 
Slit Tears.-A total of 8 slit tears was observed in 7 non-myopes (6 males, 1 female). Their numbers were few and unimportant.

In myopes a total of 39 such tears was observed in 24 eyes ( 15 males, 9 females). The estimated number of tears for each of the seven areas was as follows:

$\begin{array}{llcccccc}\text { Area } & 1 & 2 & 3 & 4 & 5 & 6 & 7 \\ \text { Number of tears (estimated) } & 5 & 28 \cdot 8 & 11 \cdot 2 & 2 \cdot 1 & 0 & 0 & 30 \cdot 8\end{array}$

It will be seen that in Area 2 (the equatorial zone) and Area 7 (the macular region) these lesions occur most frequently, but by their scarcity they are of little significance.

\section{Discussion}

There are three principal types of retinal holes-round holes, arrow-head tears, and anterior dialyses. Let us assume, in view of their morphological characteristics, that they are separate entities and each has a different aetiology. Slit tears are rare and unimportant.

\section{Round Holes}

With regard to the meridional distribution of these lesions it has been shown that they occur most frequently on the temporal side in both myopes and non-myopes, but in the former they favour the upper retina and in the latter the lower retina. The results obtained are at variance with those given by Shapland (1932) in a series of 210 cases (68 eyes with round holes). He found a much greater predilection to hole formation in the upper temporal quadrant $(52 \cdot 2$ per cent.) (present author, 36.05 per cent. non-myopes, 35.47 per cent. myopes) and very few holes in the upper nasal quadrant (1.5 per cent.) (present author, 9.88 per cent. non-myopes, 21.51 per cent. myopes). Shapland's figures for the lower temporal quadrant and lower nasal quadrant were 30.4 per cent. and 15.9 per cent. respectively, while the present author's were 40.70 per cent. (non-myopes) and 31.98 per cent. (myopes) in the former and 13.37 per cent. (non-myopes) and 11.04 per cent. (myopes) in the latter case. It may be submitted that the present much larger series reflects more clearly the true state of affairs. With regard to the radial distribution of these lesions, in both myopes and non-myopes it has been shown that they occur most frequently in a narrow zone around the equator of the eye, though notable numbers also develop more anteriorly and also at the macula. This aspect of hole distribution has not been investigated heretofore, but is of considerable importance in the aetiology, diagnosis, and treatment of retinal detachments of this type.

The evidence adduced here regarding the distribution of these holes suggests that the majority are caused by a retinal degeneration in the region of the equator. This view is supported when these fundi are examined in more detail, for not only do the holes themselves appear in an equatorial zone concentric with the ora, but frequently a more or less complete ring of degeneration can be seen before the holes appear. From its appearance this condition is called "lattice degeneration", an interlacing network of whitish lines in the retina with the holes, when they occur, appearing in the intervening tissue.

The origin of this degeneration is unlikely to be inflammatory despite the views of Arruga (1929, 1932, 1933, 1934), Duke-Elder (1945), and Longhena (1950) on the 31 
grounds that there is frequently no pigmentation, there are no other signs of past inflammation, the lesions are continuous, and no other choroidoretinitis shows a predilection for the equatorial zone. Further, patches of choroidoretinitis are more likely to produce a choroidoretinal adhesion than a hole. Indeed, this is the main purpose of surface diathermy in the treatment of detachment.

Trauma resulting in commotio retinae may be a possible cause. However, this condition does not favour the zone of the retina in question when seen clinically at the time of injury, and evidence has been adduced elsewhere (Tulloh, 1961) which is against a traumatic aetiology.

It would appear, therefore, that the condition must be a primary degeneration, and in view of the age distribution of detachments of this type, and their frequent occurrence in myopes, it may be postulated that the origin of the degeneration is vascular atrophy. Let us examine the evidence in favour of such a hypothesis.

Since the changes seen clinically must originate in the inner retinal layers (DukeElder, 1945), it is more likely to be the retinal rather than the choroidal circulation that is involved.

There is some evidence that the equatorial circulation differs from that of other parts of the retina. Wolff (1954) points out that in the equatorial zone of the retina the basic two-layered pattern of the retinal capillaries is most marked, while at about this point the deep layer disappears entirely. In addition, it will be recalled that the whole of the anterior retina as far back as the equator may derive its nutrition from the uveal circulation (anterior ciliary vessels) under certain circumstances (Duke-Elder, 1945). Herein lies a possible explanation for a relatively avascular zone at the equator-a region of the retina where the retinal blood supply becomes less efficient and where the uveal circulation is unable to augment it when senile or myopic changes supervene. In other words, a watershed, where the retina primarily supplied by retinal vessels becomes continuous with that, the nutrition of which is largely derived from uveal vessels.

The observations of Schulte (1948) support this view. He described 177 cases of detachment, of which 92 showed circulatory defects and one-third were arteriosclerotic. Knapp (1943) and Alfonso (1957), the latter from histopathological observations, also regard vascular degeneration as of considerable significance in this type of detachment, while Bonavolontà (1953), investigating 280 cases of detachment, found a frequent topographical coincidence of retinal haemorrhages and other vascular changes with retinal holes, sufficient to suggest a pathogenic relationship.

On the other hand, Michaelson (1954) although agreeing with the vascular hypothesis, considers the choroidal circulation to be primarily at fault. According to his theory, a sclerosis of the choriocapillaris occurs initially and this is followed by a retinal phlebosclerosis in the overlying tissue. Cystic degeneration and retinal hole formation follow. Such lesions were observed in the majority of the 10 cases examined about $5 \mathrm{~mm}$. from the ora. Apparently pigment migration into the retina occurred in some of these cases, though this is not a common phenomenon in the majority of early detachments.

It is more probable that the explanation of the lesions described lies in a primary retinal phlebosclerosis with varying degrees of occlusive phenomena, leading to lowgrade chronic oedematous imbibition of the surrounding retina and destruction of 
retinal elements with hole formation. Cystic changes might occur as a transient phenomenon. The preponderance of holes on the temporal side may be explained on the grounds that this is the least developed and most stretched zone of the retina, and undergoes the most marked cystoid degeneration in senile and myopic eyes (Duke-Elder, 1945).

It is suggested that the myopes acquire holes in the upper rather than the lower retina from the action of the vitreous. Vitreous bands develop in the region of the round degenerate areas which precede holes, as with arrow-head tears and presumably for the same reasons. In myopes, vitreous detachment is a frequent occurrence, particularly in the upper vitreous, because of gravity. Should the vitreous be attached by a band to the retina the pull of the formed vitreous on the retina will cause a hole to develop.

That peripheral cystic degeneration occurs in senile and myopic eyes has long been known (as Blessig's cysts) and is still thought to be causative in many detachments (Duke-Elder, 1945; Bagley, 1948; Leffertstra, 1948). Its mechanism is thought to be vascular inadequacy. However, the evidence which has been adduced here suggests that lesions (such as these cysts) at the extreme periphery are not the cause of detachment, but rather lesions at the equator. Their origin, however, may be similar, namely, vascular inadequacy.

Katzin (1951) has put forward an interesting hypothesis, that the first change is in the retina where cellular proliferation of congenital rosettes leads to cystoid degeneration and vitreous adhesion. Separation of these cells may produce an operculum and a round hole. The theory is intriguing but has not been supported by further evidence, and in any case could not account for the majority of round holes in view of the peculiar nature of their distribution.

We may conclude our discussion of the aetiology of the round holes with the hypothesis that these holes are caused by an equatorial degeneration, initiated by vascular inadequacy resulting from senile or presenile retinal arteriosclerosis, or myopia in an area where the retina is peculiarly susceptible to such changes.

The significance of this finding with regard to the diagnosis of detachments of this type lies in the necessity for the detailed examination of the whole of the equatorial region, not only for holes but also for thin and degenerate areas, and not only of the eye with the detachment but also the other eye. In myopes, holes can be expected more often above, and in non-myopes more often below, while in both cases the temporal retina is more often affected than the nasal.

Hitherto the extreme periphery has been regarded as the most frequent site for retinal holes, but it has been shown here that this is not so.

Macular Holes.-It has been shown that macular holes, usually round but occasionally slit-shaped, occur relatively frequently considering the size of the area involved. Clinically, they may be divided into two types, primary and secondary, but these two types do not differ morphologically.

Primary holes are so designated when they are the sole apparent cause of the detachment. Secondary holes, on the other hand, are produced in a long-detached macular region with holes elsewhere in the retina. Macular holes not associated with retinal detachment but resulting from macular oedema (as in central serous retinopathy or trauma) do not concern us. 
Staz (1948) considers that macular holes are a great rarity and seldom require treatment, but Cattaneo (1956) found that 4 per cent. of retinal detachments have these lesions, though he does not distinguish between primary and secondary holes. He describes 16 such cases.

Thomas (1947) and de Vincentiis (1956) distinguish primary and secondary holes, while Keeney (1955) gives theories as to their origin, either traumatic, inflammatory, or degenerative. Inflammatory holes do not concern us and traumatic holes (following Berlin's oedema) rarely give rise to detachment. Degenerative holes of vascular origin begin as cysts, according to this author, and may develop into holes later. He feels that secondary macular holes rarely occur, a view with which the present writer cannot agree.

Let us deal with secondary holes first. These must be caused either by cystic degeneration of the macula with bursting of the cyst, or, alternatively, by tearing of the retina at the fovea because of its thinness. In view of the frequency with which cystic degeneration of the retina at the macula is seen, it is probable that the cystic change is the cause of hole formation. It is tempting to suggest, however, that round holes result from cysts and slit-like holes from tears, though no proof of this can be afforded.

Primary holes, on the other hand, probably develop as a result of arteriosclerotic or myopic vascular inadequacy of the chorio-capillaris. The thinness of the retina, together with the fact that the foveal blood supply is derived solely from the choriocapillaris are predisposing factors. It is possible, however, that the same consideration may apply to macular holes as to equatorial holes in this respect, namely, the susceptibility to degenerative changes of a relatively avascular zone at the junction of an area of retina supplied by the choroidal circulation with that supplied by the retinal circulation. If this were so, the aetiology of such lesions at both the macula and equator would be the same. It is more likely, however, that at the macula the choroidal circulation alone is to blame.

The significance of macular holes lies largely in remembering to look for them and in the special techniques required for their treatment.

\section{Arrow-head Tears}

With regard to the meridional distribution of these lesions it has been shown that they occur preferentially in the upper half and temporal half of the retina in myopes, and probably in non-myopes also, though this is not proven. The proportion of tears in the upper temporal quadrant, however (40.54 per cent.), is not by any means as high as that reported by Shapland (64 per cent.) in 1932. It may be submitted that the present much larger series reflects more clearly the true state of affairs. Shapland's figures for the other retinal quadrants were as follows: Lower temporal quadrant, 2 per cent.; lower nasal quadrant, 6 per cent.; upper nasal quadrant, 28 per cent., while the present author's figures were: 24.33 per cent. (non-myopes), 20.00 per cent. (myopes); 2.70 per cent. (non-myopes), 14.05 per cent. (myopes); 32.43 per cent. (non-myopes), and 25.41 per cent. (myopes) respectively.

Investigation of the radial distribution of tears has shown that in both myopes and non-myopes they occur most frequently in a narrow zone around the equator, though notable numbers also occur more posteriorly (and not more anteriorly as with round holes). 
The fact that many of these tears occur more posteriorly than in the equatorial zone is due to the ballooning of the detached retina at the site of the tear. Examination of the fundus then reveals a tear placed apparently more posteriorly than its site of origin from the posterior wall of the globe. Of 6 cases observed by the author with arrow-head tears and balloon detachment, in which the tears were observed to fall back to the equatorial zone post-operatively, 4 showed a tear within this zone initially, but in 2 the tears were more posterior, one of these as far back as Area 5.

Though this aspect of tear-distribution has not been investigated heretofore, Gonin (1934) suggested that there might be a predilection for the equatorial zone in his observations on 100 such tears, for he found 25 per cent. of them 4 disc diameters, and 23 per cent., 5 disc diameters from the ora.

The positioning of these tears raises the question as to whether they are due primarily to a vitreous lesion or to a retinal lesion. The clinical and anatomical appearances seem to show that they result from the traction of an adherent vitreous on a weakened area of retina. Opinion is divided as to whether the major part is played by the vitreous or the retina. If the former, it must be supposed that changes in the vitreous cause it to become adherent to the retina, a vitreous detachment ensues, and the adherent strand tears a hole in the retina. The reasons adduced for the site of the tears are that the mobility of the vitreous will be most effective by its shaking movements in the upper half of the globe on account of gravity, and that the peripheral retina has little support from the nerve-fibre layer and is therefore easily torn (Duke-Elder, 1945). Doubt is thrown on this theory by the fact that tears are most frequent, not at the extreme periphery (where the vitreous is normally adherent and the retina is thinnest), but at the equator, and in addition, tears in the lower half of the globe cannot readily be explained on the same theory. Further, it is difficult to imagine how the vitreous can develop adhesions to the retina de novo.

Let us therefore consider the hypothesis that retinal changes are the major factor. The site of predilection of these lesions, namely, the equatorial zone, is the same as that for round holes, which we have seen may be caused by vascular inadequacy, myopic or senile. It is possible that tears are caused similarly, but, if so, their different morphological characteristics must be explained.

The way in which a retinal tear develops is of significance in this respect. Clinically, one sees first of all two slit-like, thin, degenerate areas set at an acute angle with one another. These then rupture producing two slit-like holes, frequently with a retinal vein running between them at their point of contact. Detachment may occur at this point and frequently does so; there is no tearing-off of the retina by a vitreous strand at the point of junction of the slit-like tears. Where there is a vitreous strand attached to the apex of the tear it would seem that the avascular operculum has undergone a degenerative process, and this has caused its attachment to the vitreous in which a "condensation" has taken place. Vitreous strands are therefore secondary and not primary. Nevertheless, they probably still contribute to the formation of tears and account for their predilection for the upper retina, in myopes particularly, in the same way as for round holes. That is, the arrow-shaped degenerate area becomes attached to a vitreous condensation. When vitreous detachment occurs, the effect of gravity predisposes to detachment above. Strands attached to degenerate retina effect traction on the retina and a tear develops. 
It will be recalled that the retinal vessels divide dichotomously, and the hypothesis is put forward here that an arrow-head tear develops from an area of ischaemic retinal degeneration caused by the partial or total occlusion of one of the minute retinal vessels at its point of division. This ischaemic retina eventually degenerates to produce a tear.

The significance of these observations with regard to the diagnosis of detachments of this type lies in the necessity for detailed examination of the whole of the equatorial region, particularly with a view to finding multiple arrow-head tears or thin and degenerate areas of a similar shape and predisposing to these lesions.

In both myopes and non-myopes the upper temporal quadrant is most often affected, but it must not be forgotten that these lesions also quite frequently occur elsewhere.

\section{Dialyses}

The results obtained have shown that these lesions have a predilection for the lower temporal quadrant of the retina, a fact established by Shapland (1932). However, his figures in respect of 62 dialyses show a slightly greater proportion in the lower temporal quadrant (77.9 per cent.) to the virtual exclusion of the lower nasal quadrant (7.4 per cent.), compared with the present author's figures of 67.39 per cent. and 19.57 per cent. respectively.

Shapland's figures for the upper temporal quadrant and upper nasal quadrant were 8.8 per cent. and 5.9 per cent. respectively, while the present author's figures for the corresponding quadrants were 10.87 per cent. and 2.17 per cent. respectively.

It is probable that most dialyses occurring in the lower quadrants are caused by trauma superimposed on a congenital retinal weakness, possibly a congenital cyst (Joannides, 1947; Juler, 1947; Charamis, 1947; Sabbadini, 1947; Shapland, 1949), while those occurring in the upper quadrants result from major trauma alone (Shapland, 1949). Hereditary congenital retinal cysts have been noted by Hamilton (1946), Sorsby, Klein, Gann, and Siggins (1951), and Jaeger (1955).

\section{Aphakia}

We have seen that the types of retinal holes and tears in aphakic eyes are similar in their morphology and distribution to those found in phakic eyes.

Although there can be no doubt that the various operations for cataract extraction precipitate retinal detachment, it would appear that aphakic detachments bear no distinguishing features compared with phakic detachments, a view with which Longhena (1950) agrees.

\section{Conclusions}

Round retinal holes in retinal detachment show a predilection for a narrow zone around the equator. In non-myopes they occur slightly more frequently below, but in myopes, more frequently above, and in both more frequently on the temporal than on the nasal side.

The hypothesis is put forward that these lesions are caused by retinal degeneration occurring in a relatively avascular zone, at the junction of an area of retina primarily supplied by the choroidal circulation with that primarily supplied by the retinal 
circulation, where the changes leading to hole formation are precipitated by senile or myopic vascular inadequacy.

The preponderance of holes on the temporal side may be explained on the grounds that this is the least developed and most stretched zone of the retina, while it is thought that myopes acquire holes in the upper rather than the lower retina because of the action of the vitreous. Vitreous bands develop in the region of the degenerate areas which precede holes. In myopes, vitreous detachment is a frequent occurrence, particularly in the upper vitreous, because of gravity. Should the vitreous be attached by a band to the retina the pull of the formed vitreous on the retina will cause a hole to develop.

The significance of the sites of predilection which have been demonstrated lies in the necessity for detailed examination of the equatorial region, not only in the eye with the detachment, but also in the other eye of the same patient, for it is here that round holes may be expected, particularly below in non-myopes and above in myopes.

Macular holes occur uncommonly in cases of retinal detachment, in both myopes and non-myopes, but relatively frequently compared with other areas of the retina when the size of the area involved is considered. They are usually round but rarely slit-like.

Though morphologically indistinguishable, two types of holes may be described: primary, in which no other retinal hole can be found, and secondary, wherein other holes are present, and the macular lesion results from long-standing detachment with cystic degeneration.

The hypothesis is put forward that primary macular holes result from arteriosclerotic or myopic vascular inadequacy of the chorio-capillaris. However, it is possible that the same consideration may apply to macular holes as to equatorial holes in this respect, namely, the susceptibility to degenerative changes of a relatively avascular zone at the junction of an area of retina primarily supplied by the choroidal circulation with that primarily supplied by the retinal circulation.

The significance of these observations lies in the necessity for looking for macular holes in all cases where the macular region is detached. For this purpose indirect ophthalmoscopy is invaluable, particularly in myopes.

Arrow-head tears show a predilection for a narrow zone around the equator. In both myopes and non-myopes they occur most frequently in the upper temporal quadrant. Their preponderance above and on the temporal side may be attributable to the action of the vitreous in the same way as for round holes.

The hypothesis is put forward that these tears are caused by linear degeneration along the course of the minute retinal vessels, originating in either myopic or arteriosclerotic vascular inadequacy, and resulting from an occlusion at the point of dichotomous division of these vessels. The intervening retina may undergo an ischaemic necrosis and, thereafter, become attached to a "condensation" of the vitreous.

The significance of these observations lies in the necessity for detailed examination of the whole of the equatorial region, particularly with a view to finding multiple arrow-head degenerative areas, the precursors of tears, or, of course, tears themselves. 
Anterior dialyses show a predilection for the lower temporal quadrant of the retina. It is concluded that those occurring below are caused by trauma superimposed on a congenital retinal weakness, possibly an hereditarily determined cyst. Those occurring above usually result from direct trauma alone.

Slit-tears are rare and unimportant.

Aphakic detachments show no distinguishing features with regard to hole formation.

\section{Summary}

In 451 cases of retinal detachment 985 holes, tears, and dialyses were observed, and their meridional and radial distribution analysed statistically.

The cases were classified according to their refraction in four groups: hypermetropes and emmetropes, low myopes, high myopes, and aphakics.

All the cases were examined with the Schepens binocular ophthalmoscope, and the holes, tears, and dialyses appropriately allocated to the four retinal quadrants in order to demonstrate their meridional distribution. A method is described whereby the radial distribution of the holes and tears could be investigated.

Theories are advanced to explain the observed facts in the light of the present investigations and those of previous writers on the subject.

The significance of the results obtained is discussed.

I wish to record my thanks to the members of the consultant staff at Moorfields Eye Hospital, London, for permission to publish details referring to their cases. I am also grateful to members of the consultant staffs of other London and provincial hospitals for referring their cases to the Detachment Clinic at Moorfields.

\section{REFERENCES}

Adamantiadis, B., and Rangavi, U. (1950). Bull. Greek ophthal. Soc., 18, 186.

Adams, S. T. (1956). Arch. Ophthal. (Chicago), 55, 254.

Agarwal, L. P. (1956). Proc. All-India ophthal. Soc., 14, 1.

Alfonso, G. F. (1957). Boll. Oculist., 36, 553.

ArkIN, W. (1948). Klin. oczna, 18, no. 2, 270.

Arruga, H. (1929). Arch. oftal. Hisp.-Amer., 29, 581.

- (1932). Ibid., 32, 461, 614. (1933). Proc. XIV int. Congr. Ophthal. (Madrid), 2, 5. (1934). Klin. Mbl. Augenheilk., 93, 52.

Bagley, C. H. (1948). Amer. J. Ophthal., 31, 285.

Böck, J. (1949). Wien. klin. Wschr., 61, 672.

BonavolontÀ, G. (1953). G. ital. Oftal., 6, 524.

Brons, K. (1924). Z. Augenheilk., 54, 117.

Campos, R. (1947). Atti 36 Congr. Soc. oftal. ital. (Roma), 36, 135.

Cattaneo, D. (1956). Boll. Oculist., 35, 1029.

Charamis, J. (1947). Trans. Greek ophthal. Soc., 14, 23.

Coccius, A. (1853). "Ueber die Anwendung des Augenspiegels", pp. 125, 128. Müller, Leipzig.

Duke-Elder, W. S. (1945). "Text-book of Ophthalmology”, vol. 3. Kimpton, London.

FrEY, R. G. (1956). Klin. Mbl. Augenheilk., 128, 50.

GARKAVI, R. A. (1952). Vestn. Oftal., 31, 23.

GonIN, J. (1930). Trans. ophthal. Soc. U.K., 50, 531.

(1934). "Le décollement de la rétine". Payot, Lausanne.

Graefe, A. von (1863a). v. Graefes Arch. Ophthal., 9, part 2, pp. 88, 111.

- (1863b). Klin. Mbl. Augenheilk., 1, 49.

Hamilton, J. B. (1946). Trans. ophthal. Soc. Aust., 6, 113. 
Hruby, K. (1953). v. Graefes Arch. Ophthal., 154, 283.

(1955). Medizinische, No. 18, 667.

JAEGER, W. (1955). Klin. Mbl. Augenheilk., 127, 423.

JoANNIDES, T. (1947). Trans. Greek ophthal. Soc., 14, 78.

JULER, F. (1947). Trans. ophthal. Soc. U.K., 67, 83.

KATZIN, H. M. (1951). W. Va. med. J., 47, 325.

KeEneY, A. H. (1955). Amer. J. Ophthal., 39, 648.

KNAPP, A. (1943). Arch. Ophthal. (Chicago), 30, 770.

KRÜMMEL, H. (1951). Klin. Mbl. Augenheilk., 119, 388.

Kümmell, R. (1929). Arch. Augenheilk,, 100-101, 314.

LEFFERTSTRA, L. J. (1948). Thesis, University of Utrecht.

LIEBREICH, R. (1863). "Atlas der Ophtbalmoscopie”, p. 17. Berlin. (1885). Ibid., p. 14.

LISTER, W. (1924). Brit. J. Ophthal., 8, 1.

Longhena, L. (1950). Proc. XVI int. Congr. Ophthal. (Lond.), 2, 1158.

Michaelson, I. C. (1954). Proc. XVII int. Congr. Ophthal. (Montreal-N. Y.), 1, 392.

Posner, A. (1951). Eye, Ear, Nose, Thr. Monthly, 30, 374. (1952). Ibid., 31, 498.

Sabbadini, D. (1947). Atti 36 Congr. Soc. oftal. ital. (Roma), 36, 604.

SChulte, L. (1948). v. Graefes Arch. Ophthal., 148, 338.

SEN, K. (1948). Proc. All-India ophthal. Soc., 9, 80.

SHAPLAND, C. D. (1932). Trans. ophthal. Soc. U.K., 52, 170. (1949). Proc. roy. Soc. Med., 42, 609.

Shipman, J. S., and Luce, C. M. (1954). Proc. XVII int. Congr. Ophthal. (Montreal-N.Y.), $2,1050$.

Sorsby, A., Klein, M., GAnN, J. H., and Siggins, G. (1951). Brit. J. Ophthal., 35, 1.

Stagni, S. (1953). Ann. Ottal., 79, 41.

Staz, L. (1948). South African Medical Congress (Pretoria), 1948. S. Afr. med. J., 22, Congress Paper p. 80.

Teng, C. C., and CHI, H. H. (1957). Amer. J. Ophthal., 44, 335.

Thomas, C. (1947). Bull. Soc. Ophtal. Paris, No. 1, 94.

Tulloh, C. G. (1961). M. S. Thesis, Durham University.

VAIL, D. (1948). Trans. Amer. ophthal. Soc., 46, 58.

DE VINCENTIIS, M. (1956). Arch. Ottal., 60, 345.

VoGt, A. (1929). Klin. Mbl. Augenheilk., 82, 619.

Wadsworth, J. A. C. (1952). Trans. Amer. Acad. Ophthal. Otolaryng., 56, 370.

DE WeCKer, L., and DE JAEGer, E. (1870). "Traité des maladies du fond de l'oeil", p. 151. Delahaye, Paris.

WeVe, H. (1932). Klin. Mbl. Augenheilk., 89, 822.

WolfF, E. (1954). "Anatomy of the Eye and Orbit", 4th ed., p. 146. Lewis, London. 